\title{
INTERROGACIONES Y AFIRMACIONES ACERCA DE MAESTRAS Y MAESTROS INVESTIGADORES
}

\begin{abstract}
Resumen
Este texto pone en escena algunas problematizaciones existentes sobre la investigación educativa y pedagógica que realizan los maestros y las maestras. Se sitúan algunas tensiones que hoy se reconocen en el ámbito académico sobre la validez y el alcance de sus producciones y se precisan algunas lineas de fuerza que coadyuvan a su reconocimiento como "maestros investigadores". Las posturas y reflexiones se argumentan con la mirada empirica a trabajos de investigación realizados por maestros y maestros que han participado en proyectos convocados y apoyados por el IDEP, realizaciones que los expresan como productores de saber y conocimiento pedagógico.

Palabras clave: Maestro investigador, investigación educativa y pedagógica, producción de saber pedagógico, sujeto de saber.

\section{QUESTIONINGS AND STATEMENTS ABOUT TEACHERS AND MASTER RESEARCHERS}

Abstract

This text brings up some questionings existing upon the pedagogical and educational research that teachers carry out. It presents some tensions which are today recognized in the academic environment upon the validity and the reach of their productions and shows some lines of strength that contribute to its recognition as "researcher teachers". The positions and reflections are analyzed with the empirical look to jobs of investigation carried out by teachers who have participated in projects arranged and supported by the IDEP, achievements that show them as producers of pedagogical knowledge.
\end{abstract}

Key words: Researcher teacher, pedagogical and educational investigation, pedagogical knowledge production, knowledge subject.

* Doctora en Filosofia y Ciencias de la Educación. Profesora-investigadora de la Universidad Pedagógica Nacional, integrante del grupo Educación y Cultura Política. mmartinez@pedagogica.edu.co.

** Magister en Investigación Social. Asesor Académico del IDEP. Profesor- investigador del CINDE. jamirez@idep.edu.co

*** Texto elaborado a partir de la mirada reflexiva a los trabajos de investigación que producen los maestros y maestras. Versión complementada a la publicada en la revista Educación y Ciudad del IDEP.

Texto original recibido: 20-01-08 y aprobado: 02-04-08

Pedagogía y Saberes N. 28. Universidad Pedagógica Nacional. Facultad de Educación, 2008, pp. 53-60 


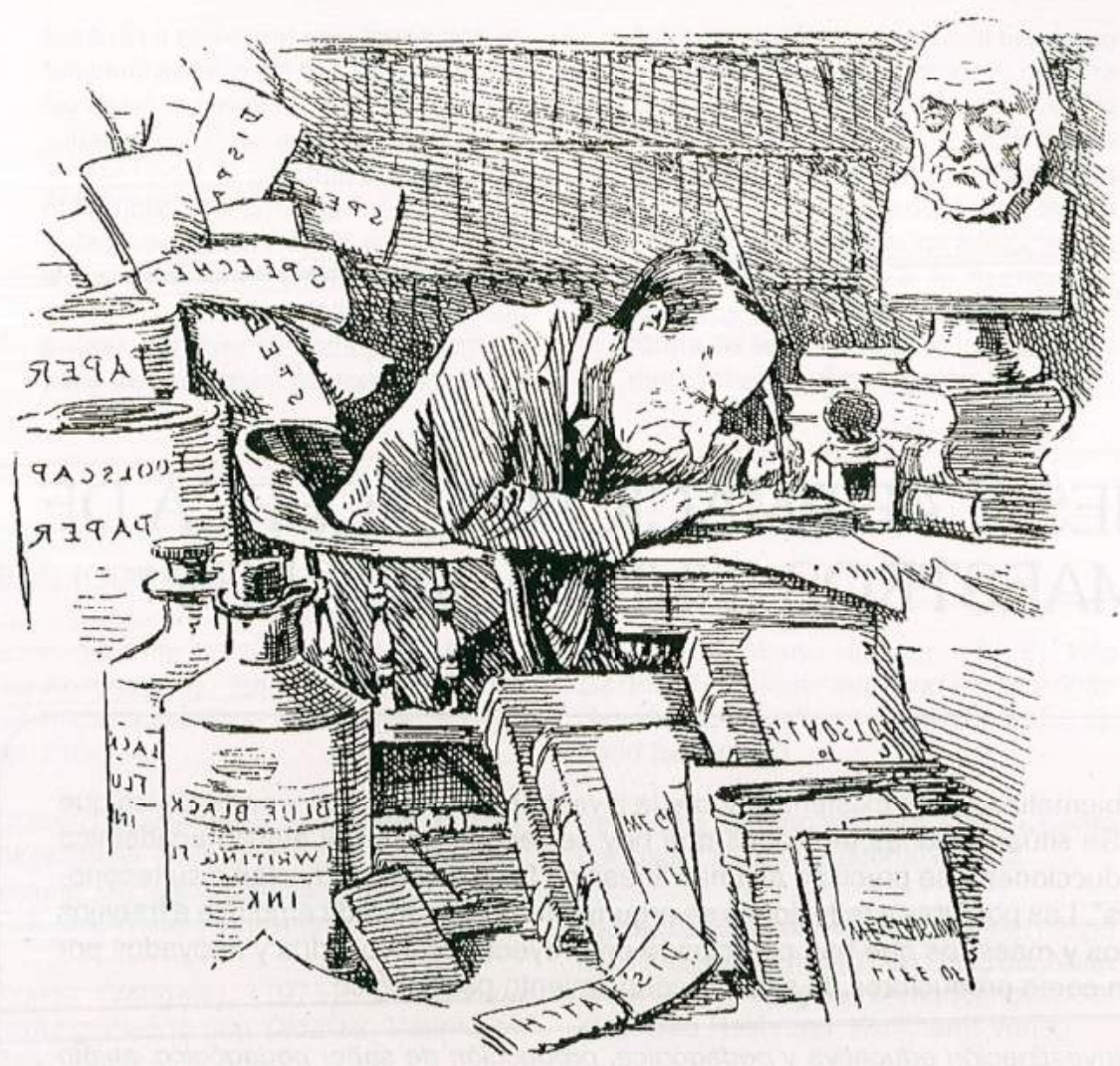

\section{TENSIONES $Y$}

\section{POSICIONES INICIALES}

Al investigar el orden social transformo el orden social y me transformo yo. La transformación que se opera en mi es la medida de la transformación que se opera en la sociedad. Como la sociedad es un conjunto autorreflexivo, puede ponerse en correspondencia con una de sus partes (que soy yo).

Un sujeto en proceso es la única medida de un proceso social.

Jesús Ibáñez

¿Cuáles son las concepciones predominantes sobre la investigación en educación y pedagogia? ¿Cómo se relacionan los maestros con la investigación en

1 IBAÑez, Jesús. (1994). Perspectivas de la investigación social: el diseño de las tres perspectivas. En Garcia, Ibáñez y Garrido. El análisis de la realidad Social: Madrid: Alianza, p. 74. educación y pedagogía? ¿Cuál es la relación y cuáles los alcances cuando se crean articulaciones entre las prácticas educativas y pedagógicas y la investigación pedagógica? ¿Acaso son incompatibles las prácticas pedagógicas con las prácticas investigativas? ¿Cuál es la noción de maestro que tienen aquellos que afirman tal incompatibilidad? ¿Cómo se ha posicionado la expresión maestro-investigador? ¿En qué escenarios investigan los maestros y las maestras?

Estos y otros interrogantes hacen parte de las discusiones y reflexiones de este texto, lo cual amerita ponerse en la agenda del debate entre las comunidades de maestros, grupos de investigación y facultades de educación.

Partimos de reconocer que es usual encontrarnos con la tensión entre las prácticas educativas y pedagógicas y la pràctica investigativa. Tensión irresoluta para un buen grupo de investigadores cuando se trata de reconocer la investigación educativa y pedagógica adelantada por los educadores y las educadoras, pero que en el caso de otras tradiciones investigativas y de algunos formadores de maestros se ve como una acción necesaria e inherente a su propio quehacer y como camino válido para la producción de saber $y$ conocimiento pedagógico que genere y agencie transformaciones educativas y pedagógicas.

Frente a la consideración de si los educadores y las educadoras de educación preescolar, básica y media hacen investigación educativa y pedagógica, podriamos situar al menos tres posiciones:

La primera plantea que las investigaciones que realizan los maestros y las maestras aluden a simples ejercicios de indagación en los que se hace uso de ciertas herramientas propias de la investigación, pero sus hallazgos no comportan un estatuto de "cientificidad". Sobre esta posición se crea una tensión que desde nuestro punto de vista está sesgada por una mirada univoca de la investigación y que nos convoca a preguntarnos sobre la noción, el sentido y el alcance de la investigación educativa y pedagógica. ¿Acaso existe una única modalidad para hacer investigación o se esperan resultados homogéneos? $\mathrm{O}$, por el contrario, ¿será que podemos hablar de niveles y modalidades de investigación $y$, por tanto, de diferencias en los hallazgos y producciones?

Una segunda posición considera que la tensión en este doble ejercicio: desempeñarse como maestro e incorporar a su rol una capacidad y práctica investigativa, deriva en una serie de acciones que amenazan el oficio del maestro en beneficio del ejercicio investigativo. Quienes la asumen sostienen que esta posibilidad conduce a la deserción del maestro del aula, algo asi como perder un buen maestro y ganar un aprendiz de investigación. Postura que podemos catalogar de egoista porque niega el devenir del maestro como sujeto productor de saber y conocimiento pedagógico y porque lo condena a actuar como subordinado -reproductor-del conocimiento producido. 
La tercera, que desde nuestra mirada viene incrementándose y busca posicionarse cada vez con más fuerza, plantea que los maestros y las maestras en ejercicio están convirtiéndose en sujetos productores de saber pedagógico, cuyo fundamento es sin duda la investigación pedagógica. En esta posición, docencia e investigación crean una acción sinérgica y de complementariedad que cualifican las prácticas educativas y pedagógicas de los educadores y la de su entorno. Cualificación que se expresa en sus acciones: son maestros que indagan, cuestionan y amplian los sentidos de sus prácticas educativas al interrogarse sobre el qué, el porqué y el para qué de lo que enseñan; sobre el sentido y la pertinencia de los contenidos objeto de aprendizaje con los contextos y las demandas de transformación; por la manera en que la escuela se convierte en creadora y constructora de cultura ética y democrática, entre otros cuestionamientos.

A esta última posición, que imbrica directamente actitud y acción investigativa al quehacer de los maestros de aula, adherimos, porque está directamente relacionada con la transformación de las prácticas educativas y pedagógicas, porque creemos que es desde la investigación, desde su episteme y razón fundante como generadora de los actos creadores, que es posible provocar transformaciones simultáneas en el sujeto y en los objetos de conocimiento.

En esta postura, los educadores organizados en grupos, colectivos y redes adelantan acciones de problematización, reflexión analitica y transformadora de sus prácticas, instauran procesos de sistematización de experiencias, acciones investigativas que construyen y reconstruyen saber pedagógico.

Hasta aqui podemos decir que mientras para algunos el rol fundamental del maestro es el de enseñante, portador de saberes disciplinares, en el que su oficio central es el de transmitir lo establecido, por tanto, desprovistos de toda actividad investigativa, para otros, el reconocimiento del maestro como productor de saber pedagógico está cruzado por la experiencia investigativa, la cual se convierte en herramienta y acción inherente a su quehacer y motor de su identidad como sujeto pedagógico.

También queda planteada la tensión sobre la postura de algunos académicos e investigadores que amparados en reglas de la investigación formal y en criterios institucionales mantienen consideraciones peyorativas que subvaloran y descalifican la investigación adelantada por maestros y maestras. Los argumentos que exponen son ya bien conocidos pero no reconocidos: falta de rigor y confiabilidad, posibilidad de hacer generalizaciones, debilidad de producción escrita por el énfasis narrativo o por los excesos anecdóticos en la recuperación de las experiencias, entre otros. Posibles inconsistencias que pueden dirimirse si se sitúa el problema en otra dimensión y se ve la investigación como una acción y condición necesaria para cualificar y transformar los procesos educativos y pedagógicos, y se reconocen los educadores como sujetos protagónicos de dicha transformación. Visión que ayudaria a bajar del pedestal la "investigación educativa y pedagógica", como si fuese privilegio de pocos, y pensarla pluralmente, con niveles $y$ modalidades de ejecución.

\section{¿DESDE DÓNDE SITUAMOS LA INVESTIGACIÓN EDUCATIVA Y PEDAGÓGICA QUE ADELANTAN MAESTROS Y MAESTRAS?}

Tratar de responder esta pregunta nos coloca frente a una perspectiva de investigación y de conocimiento que entrecruza posturas epistemológicas. metodológicas y sociopoliticas dificiles de eludir, porque se pone de presente la vieja discusión sobre la pretendida neutralidad de la investigación y el conocimiento, justificado bajo condiciones de objetividad y cientificidad.

Una especie de dictadura de la razón que se impone como acto de fe, como verdad que está por encima de la diversidad de saberes y certezas sociales construidas histórica y culturalmente. En este ámbito advierte Todorov:
Podrian coexistir varias concepciones del bien. Pero no ocurre así con los resultados de la ciencia. Aqui lo falso es implacablemente apartado y nadie piensa en pedir algo más de tolerancia para las hipótesis rechazadas. Como no hay lugar para varias concepciones de lo cierto, apelar al pluralismo no es procedente: solo los errores son múltiples; la verdad, por su parte, es una. Si el ideal es el producto de una demostración y no de una opinión, hay que aceptarlo sin protestar ${ }^{2}$.

Ese imperio del cientificismo y de la razón única, ya resuelto en el campo de las ciencias sociales y de la investigación cualitativa, coloca en el trasfondo de las tensiones anteriores el problema de la validez y legitimidad del conocimiento producido por maestros y maestras en las investigaciones que adelantan y obliga, antes de juzgarlas, a precisar el qué, por qué, para qué, con quién, cómo, dónde y desde dónde hacen investigación educativa y pedagógica.

Para situar el desde dónde asumimos nuestra posición, exponemos algunas ideas fuerza que se conectan directamente con el interrogante que nos ocupa:

La investigación educativa y pedagógica es una acción humana que posibilita la constitución de sujeto pedagógico

La constitución de sujeto pedagógico está dada por la capacidad y posibilidad del maestro-investigador para dar cuenta de algunos asuntos que le son relevantes en el campo de las prácticas educativas y en el ámbito de la reflexión sobre los saberes pedagógicos.

Instalada la investigación como una acción permanente e inherente en el maestro que asume la práctica educativa como objeto de reflexión-indagación, se despliega en este su capacidad de

2 Todorov, Tzvetan. (2002). Memoria del mal, tentación del bien. Indagaciones sobre el siglo XX. Trad. Manuel Serrat Crespo. Barcelona: Peninsula, p. 33. 
reflexión, observación y autoobservación en función de la comprensión y ampliación de su experiencia pedagógica y de su subjetividad. En este quehacer investigativo, el sujeto que investiga potencia y desarrolla actitudes para explorar, indagar, describir, reconocer, analizar, contrastar, crear, criticar, comunicar. Acciones y reflexiones que crean una dinámica de diálogo, generan intercambio de aprendizajes y una relación no escindida entre investigar y ser maestro porque producen, simultáneamente, transformación en las prácticas $\mathrm{y}$ en el sujeto.

Retomando el epigrafe de Jesús Ibáñez, porque "al investigar el orden social transformo el orden social y me transformo yo. La transformación que se opera en mi es la medida de la transformación que se opera en la sociedad. Como la sociedad es un conjunto autorreflexivo, puede ponerse en correspondencia con una de sus partes (que soy yo). Un sujeto en proceso es la única medida de un proceso social" 3 .

Sin duda, al hacer investigación se fortalecen y potencian las capacidades de observación, auto-observación, análisis, explicación, crítica, argumentación, que no se construyen teóricamente, sino en el seno de las reflexiones y comprensiones acerca de las transformaciones de la cultura, el conocimiento, la experiencia y la percepción4. La investigación provoca caminos de comprensión y de la explicación de los problemas y de la propia experiencia del sujeto en sintonia cada vez más con saberes complejos y fronterizos provenientes de la comunicación, el lenguaje, la filosofia, la sociología, la antropologia, la cultura, la politica, y cada vez menos con los saberes disciplinares tradicionales y fragmentados.

Hasta aqui afirmamos la premisa planteada: abordar la investigación educativa y pedagógica como una acción humana desarrollada también por

IBANEEZ, Jesús. Op. cit., p. 74

4 Hernandez, Carlos A. (2005). Un vinculo vital: pedagogia e Investigación. Navegaciones. El magisterio y la investigación. Bogotá: UNESCO-COLCIENCIAS. maestros y maestras no puede ser privilegio de unos pocos. La invitación es a generar más apertura para entender que la experiencia investigativa del sujeto, en este caso del maestro o maestra, es una posibilidad de configurarse creador y productor porque es en el mismo ejercicio de hacer investigación donde va constituyéndose la identidad del maestro-investigador.

La educación y la pedagogía reconocen la pluralidad de formas y niveles de hacer investigación y de producir conocimiento

Asumir el reto de comprender y explicar la experiencia pedagógica implica para el maestro un viaje, un recorrido por la razón y la emoción, no ajeno al goce y al dolor, que tiene como punto de partida un saber que se evidencia al descubrir y nombrar los sentidos otorgados a la acción y a los significados sociales construidos sobre la misma. Se trata de un recorrido por el conocimiento pedagógico. Le exige, igualmente, explicar la gramática que articula saberes, sujetos, relaciones y escenarios en la trama educativa.

Esta ruta define un lugar para la investigación educativa pedagógica, como la construcción de una narrativa que da cuenta de los sentidos y significados del campo y que se expresa en la producción de saber y conocimiento educativo y pedagógico.

En este recorrido se abre la posibilidad del diálogo crítico entre los diferentes enfoques (etnografia educativa, investigación-acción, sistematización de experiencias, construcción de memoria educativa y pedagógica, estudios históricos, entre otros), asi como entre niveles distintos de producción de conocimiento pedagógico, según los intereses, escenarios, alcances, posibilidades y condiciones. Con esto afirmamos que no hay una sola modalidad ni un solo nivel de producción de conocimiento. En esa pluralidad y diversidad de enfoques y modos de hacer investigación, vale la pena ahondar en el debate con las comunidades académicas establecidas en relación con el reconocimiento y estatus de la investigación educativa y pedagógica adelantada por maestros y maestras, clarificando que esta producción puede estar en uno $u$ otro nivel de profundización y que sus hallazgos pueden también convertirse en nuevos problemas de conocimiento.

Sin duda, es el hacer investigativo y reflexivo el que hace al maestro sujeto productor de saber y de conocimiento pedagógico y el que lo distancia del rol de consumidor y reproductor de discursos ajenos, de palabras de otros, como si ese fuera su destino sobre la tierra.

Por la investigación el maestro amplia, profundiza y transforma su mirada sobre la educación y la pedagogia

¿Qué es lo que hace al sujeto investigador? Esta es quizá la pregunta más importante a la hora de abordar el camino. ¿Es el método? ¿Son las herramientas? ¿Es el enfoque? ¿Es el proyecto? Es quizá todo y más de lo enunciado en estos interrogantes. Creemos que estos cuestionamientos se disuelven en la mirada y en la precisión del para qué se investiga.

Profundizar la mirada en un problema u objeto de conocimiento posibilita ir más allá de marchar con la corriente, de seguir huellas. Significa dejarse asombrar por lo no previsto, atreverse a pensar lo impensado, darle lugar a la imaginación $y$ a la incertidumbre como campo fértil para la creación de lo inédito. Abrirse a una nueva forma de lectura desde la que se descubren o desentrañan otras claves para pensar y actuar potencia en el sujeto sus capacidades para crear articulaciones y rearticulaciones, contextualizar aquello que enseña, crear y recrear herramientas metodológicas que lo ayuden a dar forma a los contenidos a nombrar y significar de otro modo.

Los maestros y las maestras al atreverse a investigar amplian su mirada de mundo, reinventan su práctica y sus entornos. Múltiples son los ejemplos que podriamos citar, basta evocar a los maestros del movimiento pedagógico, a los expedicionarios, a aquellos 


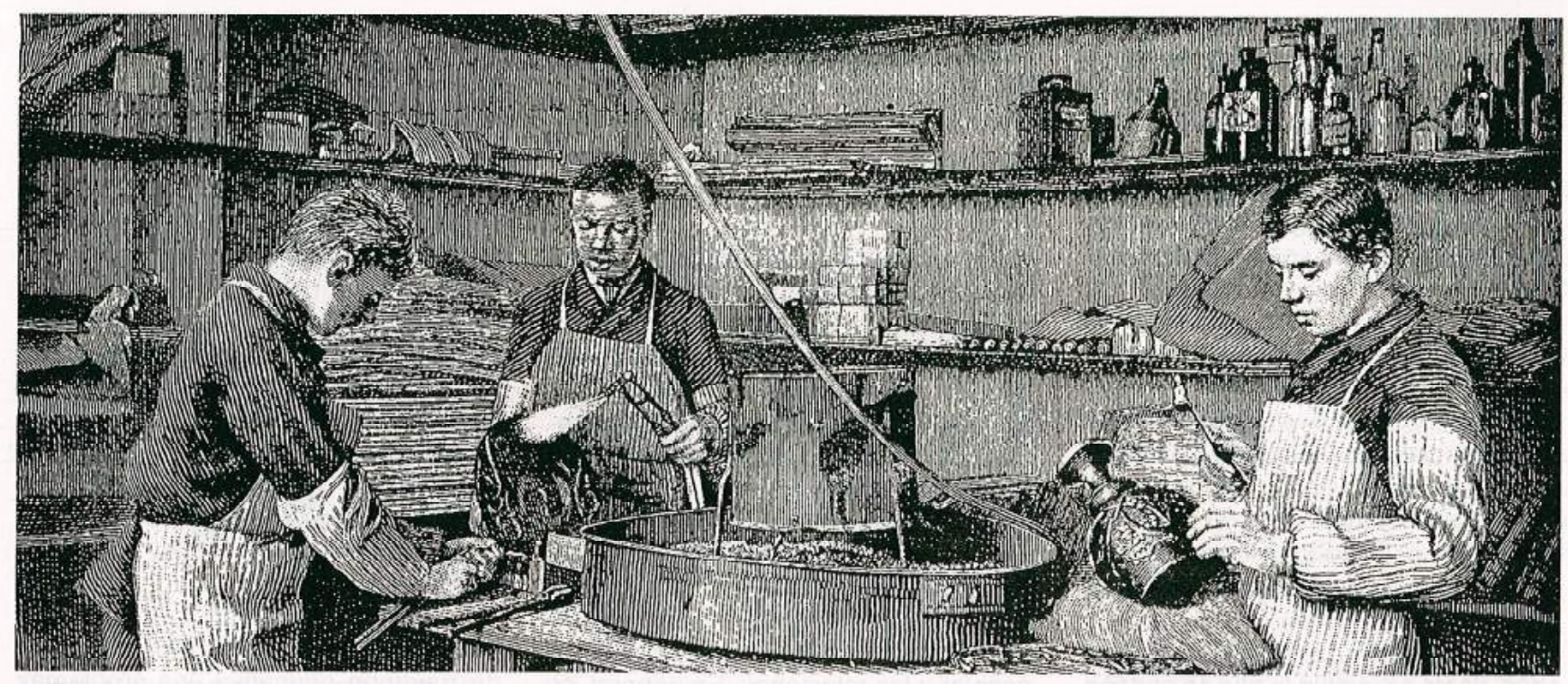

que participan en redes y colectivos; son maestros y maestras que están transformando la escuela por dentro y por fuera y nos han hecho evidente la pluralidad de pedagogias. Mientras algunos nos debatimos entre estándares y competencias, otros maestros investigan -con los rituales establecidos o más allá de ellos-, realizan experiencias de frontera y reinventan los modos de hacer escuela.

Se trata de maestros que ya han instituido la investigación como una acción cotidiana y provocan acontecimientos teñidos de movimiento pedagógico. ¿Quién decide entonces si es o no válida y legitima la investigación que realizan los maestros, si ya está produciendo efectos transformadores?

\section{Nuestra apuesta por el maestro investigador es fundamentalmente por su constitución como sujeto productor de saber y como sujeto político}

La comprensión y la explicación de la experiencia educativa y de los contextos en que esta se despliega concretan la noción de maestro como productor de saber pedagógico. Pero este saber y el proceso para producirlo tienen implicaciones que superan el simple ejercicio de conocer.
El conocimiento pedagógico es discurso y acción; por tanto, comporta una dimensión politica, ética y metodológica. La construcción de conocimiento pedagógico hace al maestro sujeto político en tanto actúa como intelectual transformativo ${ }^{5}$ y como sujeto de acción pública y politica. Esto es, como sujeto comprometido con la formación integral de sus estudiantes (hecho que supera el curriculo establecido), situado en un contexto social cambiante y corresponsable de la transformación socioeducativa de dichos contextos y con capacidades para incidir en dichas transformaciones, bien sea provocándolas o creándolas.

Al referir al maestro como sujeto político y como investigador no pretendemos distanciarlo de su realidad y condición de educador; su ejercicio y acción politica suceden en tanto se afirma como pedagogo y hace de su propio territorio -aula y escuela y entorno- el escenario propicio para las transformaciones educativas y pedagógicas. Esta es una de las razones que aducimos para reiterar la necesidad de crear condiciones para la constitución de maestros y maestras investigadoras.

5 GiRoux, Henry (1990). Los profesores como intelectuales. Hacia una pedagogía critica del aprendizaje. Barcelona: Paidós.

\section{¿SOBRE QUÉ INVESTIGAN LOS MAESTROS $Y$ LAS MAESTRAS?}

¿Qué asuntos, qué problemas y en qué escenarios emerge la investigación de los maestros y las maestras? ¿Qué alcances podemos precisar de las investigaciones que realizan? Para evitar reflexiones especulativas, documentamos las afirmaciones realizadas con la información empirica que ofrece el IDEP ${ }^{6}$ acerca de los asuntos y pro-

6 Cfr. Las fuentes de esta indagación:

- Reporte de investigaciones y de experiencias educativas 2000-2006 apoyadas por el IDEP. Centro de Memoria del IDEP

- Camargo Abella, Marina y Ramirez, Jorge $\mathrm{E}$. El desarrollo de la investigación y la innovación educativa durante el trieno 1998-2001. Evaluación de resultados de los proyectos de inversión: Investigaciones para cualificar el desempeño y la formación de docentes, y Fomento, investigación y socialización de las innovaciones educativas. Bogotá, 2001.

- Camargo Abella, Marina, Calvo, Gloria, y otras. (2005). Informe de investigación: El estado del arte de la investigación educativa y pedagógica 2000-2004. Instituto de Investigación Educativa y Desarrollo Pedagógico. IDEP. Bogotá. Mimeo. 
blemas que investigan los maestros vinculados a los proyectos del Instituto, aclarando que esta mirada no agota el universo de esta problemática.

Para abordarlos dividimos los asuntos sobre los que investigan en dos campos: los pedagógicos y los educativos.

\section{Los asuntos pedagógicos}

Las investigaciones referidas a las actividades de aula pueden agruparse en tres. Un primer grupo da cuenta de problemas orientados a mejorar, cualificar o transformar las relaciones y procesos de enseñanza y aprendizaje. Se hacen preguntas referidas al qué, por qué, para qué, cuándo, para quién y cómo se enseña, cómo se evalúa lo que se enseña. Otro grupo de preguntas se relacionan con el curriculo y el plan de estudios. Con menor frecuencia se pregunta por las relaciones entre aquello que se enseña y lo que se aprende y por los efectos e impactos de las prácticas.

Un segundo grupo asume temas-problema "transversales" al curriculo, como la lectura y la escritura, la educación sexual, el espiritu cientifico, la comunicación, la informática, la educación especial, la lúdica y el juego. Estas investigaciones se orientan a construir nuevos tipos de experiencias por fuera de las fronteras del conocimiento disciplinar complejizando, en parte, las prácticas curriculares.

El tercer grupo asume problemas referidos a los ambientes educativos y de aprendizaje, que aunque están muy relacionados con los anteriores "conducen a la elaboración de sentidos, ideas, afectos, acciones y significados"7 de las prácticas. Indagan por los contextos inmediatos y las condiciones de orden social, cultural, tecnológico, espacial, por el tipo de relaciones que establecen $y$ las maneras en que estas inciden en

7 Escuela Pedagógica Experimental-IDEP (1999). La construcción de la confianza Una experiencia en proyectos de aula. Bogotá, p. 33. la enseñanza y en los aprendizajes. Se abordan temas como los valores de la participación, el trabajo en equipo, el conflicto en el aula, ambientes educacionales, medios de enseñanza y solución de problemas.

La aproximación investigativa y tratamiento metodológico de este tipo de problemas, en los tres casos, se hace desde enfoques cualitativos de investigación, preferenciando la etnografia, los estudios de caso y la sistematización de experiencias como modalidad investigativa. En su conjunto buscan producir conocimiento pedagógico sobre la enseñanza de los conocimientos disciplinares o transdisciplinares orientados a afectar y enriquecer las prácticas pedagógicas, el curriculo, el ambiente escolar y las relaciones. En general, se observa un amplio énfasis didáctico y metodológico.

\section{Los asuntos de investigación educativa}

Partimos de reconocer que el campo de la investigación educativa abarca la relación educación-sujeto-sociedad y en esa medida exige miradas interdisciplinarias.

Las investigaciones referidas a los asuntos educativos institucionales en escenarios que trascienden las fronteras del aula incluyen problemas institucionales, del entorno, de las condiciones sociales, situaciones que por lo general no son valoradas cuando se evalúa el quehacer del maestro.

Se ubican es este campo las investigaciones adelantadas por los maestros y las maestras que utilizando diferentes referentes conceptuales y herramientas metodológicas logran elaborar y ampliar comprensiones de contexto para la acción educativa, reconocer el alcance de su participación en la formulación de politicas educativas institucionales o locales y la importancia de asumir posturas criticas y propositivas que contribuyan a reorientar o complementar los sentidos de la acción educativa y pedagógica que adelantan.
Se trata de investigaciones localizadas en un ámbito institucional escolar o local que abordan problemas como: los estudios diagnósticos de la situación educativa en el colegio y sus relaciones con los proyectos educativos institucionales, $\mathrm{PEI}$, y los planes locales de educación, el estado de los $\mathrm{PEI}$, la formación para los derechos humanos y la ciudadania, las prácticas de convivencia y solución negociada del conflicto escolar, la comunicación y los medios masivos, la atención pedagógica a poblaciones vulnerables (discriminación por género, por situación de desplazamiento forzado, por necesidades educativas especiales), la participación comunitaria y relaciones escuela-comunidad, la formación de maestros en los ámbitos institucional y local, la recuperación y construcción de memoria educativa, los problemas de deserción y permanencia escolar, los asuntos de gestión escolar y participación comunitaria, representaciones sociales que circulan en la escuela sobre diversos asuntos (sexualidad, democracia, conflicto, convivencia, aprendizaje, enseñanza, evaluación, entre otros).

Sin duda, la vinculación y relación de los maestros y las maestras con estos temas y el conocimiento pedagógico que producen estas investigaciones generan formas de acercamiento que extrapolan y derrumban las fronteras de la escuela, multiplican los espacios de interacción e indagación, vinculan a otros actores educativos, demandan la interdisciplinariedad de saberes y amplian las posibilidades de aprendizajes descentrándolos de las dinámicas de la enseñanza. Aqui son evidentes un enriquecimiento y una complejización del saber pedagógico.

Como en los asuntos pedagógicos, se trata de estudios de corte cualitativo con diferentes modalidades y herramientas. Hecho que señala y da cuenta del acercamiento y la estrecha relación que establecen los maestros y las maestras que investigan, con las lógicas, presupuestos y dinámicas propias de la investigación educativa y pedagógica. También de la ampliación del saber pedagógico a una dimensión social y educativa. 


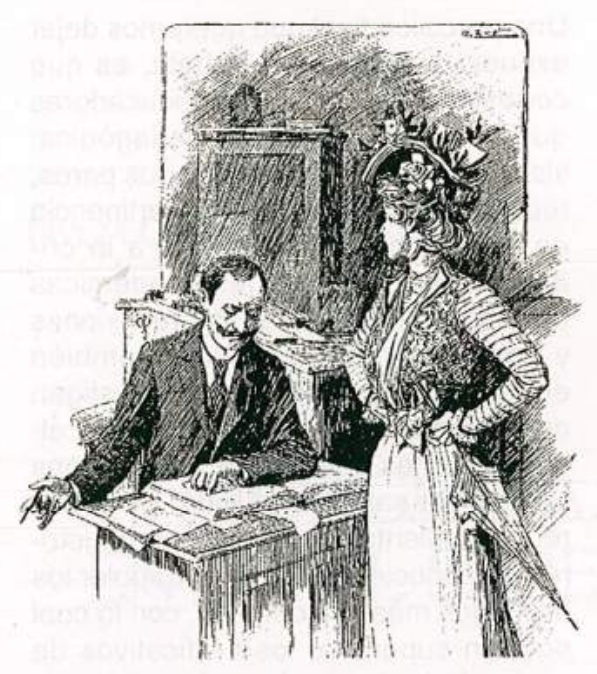

¿QUÉ CARACTERISTICAS Y
CONCLUSIONES SE DERIVAN
DE ESTAS INVESTIGACIONES?

El estado del arte $(2000-2004)^{8}$ de la investigación educativa y pedagógica realizada por el IDEP señala las siguientes caracteristicas generales:

- La investigación pedagógica adelantada por los maestros abrió posibilidades a la escritura sobre el saber y su hacer especifico a partir del análisis de las prácticas de enseñanza de las áreas disciplinares y amplió el marco de comprensión de lo pedagógico.

- La investigación se realiza bajo diferentes modalidades (reflexión colectiva, sistematización de experiencias, investigación de aula) orientada al mejoramiento de la calidad de la educación.

- Los maestros analizan su propio quehacer, son autoobservadores de lo que sucede en el aula y en otros espacios educativos $y$, por tanto, aportan saberes y conocimientos a la pedagogia. Los hallazgos les permiten preguntarse por los cambios

8 Camargo Abella, Marina, Calvo, Gloria, y otras. (2005). Informe de investigación: el estado del arte de la investigación educativa y pedagógica 2000-2004. Bogotá: Instituto de Investigación Educativa y Desarrollo Pedagógico, IDEP. (en prensa). contemporáneos y su inscripción en las prácticas de enseñanza. Cuestionamientos que necesariamente cualifican su propio quehacer.

- Las indagaciones por el contexto, por lo educativo y por asuntos colaterales que inciden en el aprendizaje logran que los maestros propongan cambios y cualifiquen sus prácticas en función de las realidades socioculturales de los estudiantes.

Al tener como punto de partida la práctica y los saberes que la sustentan, se evidencia una dirección en el proceso de investigación orientada a la construcción de conocimientos pedagógicos, lo cual sugiere diversos estados de elaboración en el tránsito de la producción saberconocimiento.

En general, estas conclusiones enlazadas con nuestras propias experiencias de trabajo con grupos de investigación y redes de maestros y con las apreciaciones construidas desde la dinámica investigativa que fluye entre escuela y maestros nos ayudan a argumentar la tesis que hemos venido esbozando y que podemos sintetizar de esta manera: las investigaciones que adelantan los maestros y las maestras producen conocimiento educativo y pedagógico teóricopráctico que tiene varias finalidades:

- Ampliar la comprensión de las prácticas educativas y pedagógicas, descifrando los sentidos que los mismos maestros le otorgan y construyendo nuevos significados y modos de realización.

- Producir explicaciones críticas de estas prácticas, lo cual significa un cierto ejercicio de teorización de las mismas haciendo uso de categorias provenientes de saberes disciplinares, transdiciplinares y de "conocimientos socioculturales" contemporáneos.

- Reorientar y proyectar dinámicas y acciones de transformación en las relaciones, sujetos y escenarios que constituyen estas prácticas.

- Proponer formas alternas de pensar y hacer pedagogia, rutas conceptuales y metodológicas que materialicen las reorientaciones y proyecciones emergentes.

En este sentido y con los reconocimientos anteriores es claro que la investigación pedagógica y educativa actúa como una reconstrucción crítica del saber pedagógico y crea posibilidades para la ampliación y cualificación de la práctica pedagógica, la reflexión, construcción, intercambio y socialización de experiencias. Adicionalmente se convierte en generadora de comunidades académicas porque su ejercicio rompe la insularidad de las prácticas, compromete y convoca sujetos colectivos sin negar la diferencia y singularidad de cada sujeto individual.

\section{PRETEXTOS PARA CONTINUAR}

Evidentemente los asuntos expuestos en esta argumentación hacen un reconocimiento explicito a la validez y legitimidad de la investigación adelantada por las maestras y los maestros. Con ello también afirmamos la condición de cientificidad de la investigación, que en tanto doctrina impuesta como único criterio de verdad se convierte en cientificismo, no en referente único ni suficiente como criterios de validez y confiabilidad. Entre otras razones porque en esta condición el cientificismo no es otra cosa que un discurso ideológico construido sobre una visión de ciencia y de conocimiento que ha sido suficientemente cuestionada.

En este sentido, en la investigación social y, en particular, en las investigaciones en educación y pedagogia se hace evidente, una vez más, que se requiere tener en cuenta las posturas, intereses, delimitaciones, modalidades, tipos y niveles de producción de conocimiento que se produce, sin que se trate de implementar o validar reduccionismos o de negar el rigor y exigencia de producción que le son inherentes.

Reconocemos que la coherencia interna, la rigurosidad metodológica, la confiabilidad de la información y la secuencialidad lógica son criterios inseparables de todo proceso investigativo, pero estos no pueden imponerse, verse y evaluarse desde un solo lugar o postura, ni obrar 
como dispositivos de poder o control que atiendan solo a intereses externos. La validez y la confiabilidad, como el alcance, se construyen en la dinámica investigativa entre los maestros investigadores y se legitiman con los diversos actores educativos que en ella participan en la medida que sus contribuciones se instalen en la cotidianidad escolar.

No se trata entonces de legalizarla a partir del cumplimiento de cánones preestablecidos, ni de reducirla solo al cumplimiento de criterios formales de lógica y coherencia interna. Su validez y legitimidad están dadas en tanto sus hallazgos y productos se traducen en transformaciones de las realidades educativas y pedagógicas tomadas como objetos de investigación y en los sujetos que la producen. También hay que valorarla, más que medirla, con base en sus propósitos y alcances y

\section{BIBLIOGRAFÍA}

Camargo, Marina, Calvo, Gloria, y otras (2005). El estado del arte de la investigación educativa y pedagógica 2000 - 2004. Instituto de Investigación Educativa y Desarrollo Pedagógico - IDEP. Bogotá (En prensa).

Camargo, Marina y Ramirez, Jorge E.(2001) El Desarrollo de la investigación y la innovación educativa durante el trieno 1.9982001. Evaluación de resultados de los proyectos de inversión: "Investigaciones para cualificar el desempeño y la formación de docentes" y "Fomento, investigación y socialización de las innovaciones educativas". Bogotá: IDEP (en prensa). niveles de producción, que bien pueden ser estudios explicatorios o descriptivos que sirvan de base para otros de mayor profundización.

No se trata, por tanto, de criterios de verificación en términos de lograr indicadores de evaluación con patrones de contrastación totalmente establecidos, tampoco de buscar condiciones de generalización de los resultados desconociendo el carácter particular y local de este conocimiento. Se trata de entender y reconocer que la investigación adelantada por maestros y maestras, en particular la investigación pedagógica, es una posibilidad de reconocer el nivel profesional de los maestros y maestras $y$, por su puesto, de contribuir a la cualificación de los procesos educativos y pedagógicos que se promueven desde la escuela.

Centro de Memoria del Idep.-Reporto de investigaciones y de experiencias educativas $2.000-2.006$ apoyadas por el IDEP. (Documento de trabajo. Sin publicar).

GiRoux, Henry (1990). Los profesores como intelectuales. Hacia una pedagogía critica del aprendizaje. Barcelona: Paidós.

Hernandez, Carlos A. (2005). Un vínculo vital: Pedagogía e Investigación. Navegaciones. El magisterio y la investigación. Bogotá: UNESCO-COLCIENCIAS.

IBAÑEz, Jesús (1994) Perspectivas de la investigación social: el diseño de las tres
Una precisión final que queremos dejar expuesta y abierta al debate, es que corresponde a los mismos educadores que hacen investigación pedagógica: visibilizarla, extenderla entre sus pares, reconociendo la necesaria pertinencia en cada contexto, someterla a la critica de las comunidades académicas para que se produzcan las reflexiones y complementos necesarios también en los mismos sujetos que investigan como acción autocrítica. Estas son algunas formas de producir valoraciones acerca del saber producido, de lograr reconocimiento como sujetos productores de conocimiento y de extrapolar los hallazgos más allá del aula, con lo cual podrian superarse los calificativos de que se trata de simples esbozos investigativos autocentrados en los mismos maestros. perspectivas. En: García, Ibáñez y Garrido. El análisis de la realidad Social: Madrid: Alianza

Segura, Dino (1999). La Construcción de la confianza. Una experiencia en proyectos de aula. Bogotá Bogotá. Escuela Pedagógica Experimental - IDEP

TODOROv. Tzvetan, (2002). Memoria del mal, tentación del bien. Indagaciones sobre el siglo XX. Trad. Manuel Serrat Crespo. Península: Barcelona. 